\title{
Cognitive-behavioural therapy for anxiety in dementia: pilot randomised controlled trial
}

\author{
Aimee Spector, Georgina Charlesworth, Michael King, Miles Lattimer, Susan Sadek, \\ Louise Marston, Amritpal Rehill, Juanita Hoe, Afifa Qazi, Martin Knapp and Martin Orrell
}

\section{Background}

Anxiety is common and problematic in dementia, yet there is a lack of effective treatments.

\section{Aims}

To develop a cognitive-behavioural therapy (CBT) manual for anxiety in dementia and determine its feasibility through a randomised controlled trial.

\section{Method}

A ten-session CBT manual was developed. Participants with dementia and anxiety (and their carers) were randomly allocated to CBT plus treatment as usual (TAU) $(n=25)$ or TAU $(n=25)$. Outcome and cost measures were administered at baseline, 15 weeks and 6 months.

\section{Results}

At 15 weeks, there was an adjusted difference in anxiety (using the Rating Anxiety in Dementia scale) of $(-3.10$, $95 \% \mathrm{Cl}-6.55$ to 0.34 ) for CBT compared with TAU, which just fell short of statistical significance. There were significant improvements in depression at 15 weeks after adjustment $(-5.37,95 \% \mathrm{Cl}-9.50$ to -1.25$)$. Improvements remained significant at 6 months. CBT was cost neutral.

\section{Conclusions}

CBT was feasible (in terms of recruitment, acceptability and attrition) and effective. A fully powered RCT is now required.

\section{Declaration of interest}

None.

\section{Copyright and usage}

(c) The Royal College of Psychiatrists 2015.
Anxiety in dementia is common, with prevalence estimated from 5 to $21 \%$ for anxiety disorders and up to $71 \%$ for anxiety symptoms. ${ }^{1}$ Anxiety may physically present as motor restlessness, agitation, day/night disturbance and/or aggression, and as the dementia progresses, often results in exacerbated symptoms as a result of increased dependency and behavioural problems. ${ }^{2,3}$ Anxiety in dementia often receives little attention. ${ }^{1}$ People may get treated with psychotropic medication (such as antidepressants), even though there is little if any evidence of benefit and use of antipsychotics presents serious risks. ${ }^{4}$ Cognitive-behavioural therapy $(\mathrm{CBT})^{5}$ is a collaborative psychological approach that addresses the interaction between people's thoughts, feelings and behaviour. There is robust evidence that CBT is an effective first-line strategy for anxiety in older people without dementia. ${ }^{6}$ The UK National Health Service (NHS) widely endorses CBT through its Improving Access to Psychological Therapies (IAPT) programme, ${ }^{7}$ which supports primary care trusts in implementing CBT for depression and anxiety. There is evidence that people with dementia can learn and develop skills, ${ }^{8}$ which suggests that CBT could be used for people with dementia as it has been with other impaired populations including individuals with intellectual disabilities. ${ }^{9}$ There is some evidence for the feasibility of CBT for anxiety and depression in dementia, primarily through case studies and two small randomised controlled trials (RCTs) in the USA (for example Teri et al, Kipling et al, Paukert et al and Stanley et $\left.a l^{2,10-12}\right)$. They all concluded that larger trials are needed. This study had two phases, which correspond to phase I and II of the Medical Research Council's (MRC's) guidelines for developing a complex intervention and assessing feasibility. ${ }^{13}$ They were to: (a) develop a CBT intervention manual and (b) assess the feasibility of the intervention through a single-blind, pilot RCT of CBT plus treatment as usual (TAU) versus TAU for people with dementia (supported by their carers). This included an assessment of acceptability, adherence, recruitment, retention and costs. Ethical approval was obtained through the 'East London 3 Research
Ethics Committee' (reference number 10/H0701/124). The trial registration number is ISRCTN46521766.

\section{Method}

\section{Stage I: manual development}

The manual was developed in several stages, described previously. ${ }^{14}$ Its development involved a systematic literature review, expert review, a consensus conference with 30 people and field-testing with three people with dementia. The version used in this trial involved a three-phase formulation-driven therapy based on Beck \& Clark's ${ }^{5}$ cognitive model of anxiety, whereby an individualised (person-centred) formulation is collaboratively reached to guide progress. Phase 1 involves building a collaborative relationship, psychoeducation about CBT and the excess disability caused by anxiety in dementia, self-monitoring, developing an individualised formulation and identifying goals. During this first phase, the level of carer involvement is also established. The carer's role is to support the person with dementia in implementing strategies, for example applying what has been discussed during sessions in everyday life. Their involvement could range from very little (for example attending brief parts of some sessions) to being present at all times.

Phase 2 involves the application of change processes, which the therapist can adapt according to the needs and strengths of the individual. These include identifying and practising strategies for feeling safe, identifying and challenging unhelpful cognitions, addressing 'realistic negative automatic thoughts', calming thoughts (on cue cards) and behavioural experiments. Phase 2 also had optional 'modules' for considering longstanding unhelpful 'rules for living' and for addressing interpersonal difficulties between the carer and person with dementia. Phase 3 works on ending the therapy and developing a blueprint for the future. This includes reviewing and consolidating learned skills, 
integration of skills into everyday life and considering the future involvement of carers and others.

\section{Stage II: RCT}

Design

A single-blind, multicentre, pilot RCT of CBT plus TAU (CBT group) versus TAU (TAU group) for people with anxiety and dementia. As no trials have been done in this area, we were unable to estimate the likely effect size of this intervention. The sample size was chosen on pragmatic grounds as sufficient to demonstrate adequate recruitment and retention, although 50 participants would be sufficient to detect an effect size of 0.8 with $80 \%$ power and $5 \%$ significance. We also aimed to provide data on the possible effect size of the intervention in order to inform a power analysis for a large-scale RCT.

\section{Participants}

Participants were eligible for inclusion if they:

(a) met DSM-IV criteria ${ }^{15}$ for dementia in the mild-to-moderate range, determined by a Clinical Dementia Rating $(\mathrm{CDR})^{16}$ score of $0.5,1$ or 2 ;

(b) had clinical anxiety, as determined by a score of 11 or above on the Rating Anxiety in Dementia (RAID) scale, ${ }^{17}$ with or without comorbid depression;

(c) lived in the community;

(d) had a self-identified carer who was willing to participate in the therapy;

(e) were able to understand and communicate in English;

(f) were willing to engage in therapy involving discussion of thoughts and feelings.

Participants were excluded if they had (a) a comorbid psychiatric disorder (such as psychosis) or challenging behaviour (for example severe agitation), likely to prevent engagement in therapy or (b) the presence of an intellectual disability or severe physical illness, which could have an impact on participation.

\section{Procedure}

Potential participants were primarily identified through NHS secondary care services within two NHS trusts. People who appeared to meet inclusion criteria were contacted by telephone or in writing by the referrer. If they chose to participate, informed consent was sought from participants and their carers using current guidance from the British Psychological Society on evaluation of capacity. Following this, people were screened for suitability and the full assessment was conducted if they were deemed suitable.

\section{Assessments}

All assessments were administered by the research assistant at week 1 (baseline), week 15 (follow-up 1) and 6 months (follow-up 2). Initial field testing of the intervention revealed that it took more than 10 weeks to complete the ten sessions, because of factors such as illness and holidays. Follow-up 1 therefore took place at 15 weeks, to increase the likelihood that the intervention had been completed. Baseline data collected included age, gender, ethnic group, use of medication and participation in other activities.

The primary outcome was measured using the RAID scale. ${ }^{17}$ This rates signs and symptoms of anxiety using interviews with carers and people with dementia. There are 18 questions in four categories: worry, apprehension, vigilance, motor tension and autonomic hypersensitivity. A score of 11 or above indicates significant clinical anxiety, with a maximum possible score of 54. It has good interrater and test-retest reliability and is sensitive to change.

Costs were measured using the Clinical Services Receipt Inventory (CSRI), ${ }^{18}$ which collects information about the participant's receipt of health and social care services, equipment or adaptations, medication, accommodation (for example care home), income and benefits. Changes in the receipt of these services can be tracked over time, as it asks for service receipt over the previous 3 months each time it is administered.

Depression was measured using the Cornell Scale for Depression in Dementia (CSDD) ${ }^{19}$ This rates depression in the person with dementia in five domains including mood-related signs, behavioural disturbance and ideational disturbance, using interviews with people with dementia and proxies. Good reliability and validity have been demonstrated. The mood of both the person with dementia and their carer was also measured using the Hospital Anxiety and Depression Scale (HADS), ${ }^{20}$ a widely used measure validated for all age groups. Quality of life was measured using the Quality of Life - Alzheimer's Disease (QOL-AD), ${ }^{21}$ a self-report measure for the person with dementia and their carer, with 13 items covering domains including physical health, energy, friends and fun. It has excellent interrater reliability and internal consistency, and good content, criterion and construct validity. Behavioural disturbance was measured by the Neuropsychiatric Inventory (NPI). ${ }^{22}$ This assesses ten areas including delusions, hallucinations, dysphoria and agitation/ aggression. Content and concurrent validity, interrater and testretest reliability and internal consistency are all good. Cognitive function was assessed by the Mini-Mental State Examination (MMSE). ${ }^{23}$ This is an internationally recognised, 11-item set of simple tasks presented to the participant including orientation to time and place, attention, recall, language and visual construction. It has a maximum score of 30 points, with 24 or less suggesting cognitive impairment. Reliability and validity are satisfactory. Person-carer relationship was assessed using the Quality of Caregiver and Patient Relationship (QCPR). ${ }^{24}$ This is a 14-item scale measuring relationship quality including the level of criticism and level of warmth, rated by both the person and their carer. Good reliability and validity have been demonstrated.

\section{Randomisation procedures}

Patient-carer dyads were randomly allocated to either CBT or TAU, with an allocation ratio of 1:1. The randomisation sequence was generated using Stata version 12.1 for Windows by an independent statistician and administered by PRIMENT Clinical Trials Unit after the participant/carer had provided consent and baseline data. A method of blocking was employed (with block sizes varying between four and six) to help ensure equal numbers in the intervention and control arms. Once each individual was randomised, the Clinical Trials Unit informed the trial psychologist of allocation and the psychologist then informed the individual by telephone. Assessors were masked to group allocation. Participants with dementia and their carers could not be masked to group allocation because the intervention was psychosocial. However, they were reminded not to disclose which arm of the study they were assigned to at the beginning of each assessment, in an attempt to minimise detection bias.

\section{Intervention and control groups}

In the CBT group the patient-carer dyads participated in up to ten weekly sessions, each lasting approximately $1 \mathrm{~h}$. This number was determined on the basis of the published literature, the team's 
experience and patient and carer feedback during field testing. Sessions were shortened and breaks taken as required to maintain attention. Sessions were delivered by four clinical psychologists with experience of working with people with dementia. Participants receiving CBT were permitted to utilise any standard treatment available for anxiety if required. In the TAU group participants received the standard treatment available to people with anxiety and dementia, which was most likely to include medication or no treatment.

\section{Statistical methods}

Data were analysed using the intention-to-treat principle. Baseline summary statistics by randomised group were calculated. Outcomes at 15 weeks and 6 months were modelled separately using linear regression, with coefficients and $95 \%$ confidence intervals presented (see online supplement). Results for the participants with dementia are presented unadjusted and adjusted for baseline anxiety (score on the RAID) and baseline cognition (score on the MMSE). The sample size meant that we were unable to adjust for any other baseline covariables. Analyses on the carer scales were adjusted for the value of the scale at baseline. It was agreed $a$ priori that the adjusted analyses would be the primary analyses. All analyses were carried out using Stata version 12.1 for Windows.

\section{Cost analysis}

The cost analysis adopted a health and social care perspective, which considers only costs incurred by organisations providing health and social care services. Unit costs were obtained from the Personal Social Services Research Unit (PSSRU) compendium for $2011^{25}$ where possible. It was decided a priori that cost differences at both follow-up points would be compared after adjusting for pre-baseline health and social care costs, baseline MMSE score and baseline RAID score, using multiple regression.
To minimise the effect of skewness, $95 \%$ bias-corrected confidence intervals for between-group mean costs were estimated using non-parametric bootstrapping techniques (re-sampling with replacement; 1000 repetitions). Missing values within the main cost analysis were addressed through imputing mean values. For each variable used in the cost analysis, missing values accounted for fewer than $15 \%$ of total responses. Two sensitivity analyses were conducted. The first used no imputations (costs that could not be calculated because of missing data were excluded) and the second excluded outliers (individuals with abnormally high costs in one or more cost categories, for example accommodation costs).

\section{Results}

\section{Participants}

Tables 1 and 2 show the characteristics of the sample; Table 1 for the people with dementia and Table 2 for the carers. All participants had received a formal diagnosis of dementia. There were 20 males and 30 females aged between 63 and 98 years. Randomised groups were balanced in terms of gender, mean age and ethnicity of the patient (Table 1). In the CBT group, all carers were family members, whereas this was the case for $80 \%$ in the TAU group. This might explain the much higher median hours spent a week caring in the CBT versus the TAU group (61 v. 15), with family carers generally describing their care as $24 \mathrm{~h} 7$ days a week (Table 2). The median MMSE was 23 for both groups. However, the median RAID was lower in the CBT group compared with the TAU group (17 (IQR 14-21) v. 22 (IQR 17-24), respectively) (Table 1 ). There was also a lower use of anxiolytic medication in the CBT group $(8 \%)$ compared with the TAU group $(24 \%)$.

\section{TAU}

All participants received standard treatment, described as TAU. In total, $62 \%$ of the sample was on antidepressant medication and

\begin{tabular}{|c|c|c|c|c|c|c|}
\hline \multirow[b]{2}{*}{ Variable } & \multicolumn{3}{|c|}{ CBT group $(n=25)$} & \multicolumn{3}{|c|}{ TAU group $(n=25)$} \\
\hline & $n(\%)$ & Mean (s.d.) & Median (IQR) & $n(\%)$ & Mean (s.d.) & Median (IQR) \\
\hline \multicolumn{7}{|l|}{ Sociodemographics } \\
\hline Age & & $78(7)$ & & & $79(7)$ & \\
\hline Male & $10(40)$ & & & $10(40)$ & & \\
\hline Black or minority ethnicity & $0(0)$ & & & $1(4)$ & & \\
\hline Years in education & & & $9(9-10)$ & & & $10(9-11)$ \\
\hline Use of anxiolytic medication (lorazepam, diazepam, buspirone) & $2(8)$ & & & & $6(24)$ & \\
\hline $\begin{array}{l}\text { Use of antidepressant medication (citalopram, mirtazapine, } \\
\text { sertraline hydrochloride, paroxetine, venlafaxine, amitriptyline) }\end{array}$ & $12(48)$ & & & & $9(36)$ & \\
\hline Use of antipsychotic medication (quetiapine) & $0(0)$ & & & $2(8)$ & & \\
\hline \multicolumn{7}{|l|}{ Standardised scales } \\
\hline Mini-Mental State Examination & & $21(5)$ & $23(19-24)$ & & $20(6)$ & $23(16-25)$ \\
\hline Quality of Life Alzheimer's Disease & & $34(5)$ & $35(30-38)$ & & $35(6)$ & $34(32-38)$ \\
\hline Quality of Caregiver-Patient Relationship & & $59(5)$ & $61(57-63)$ & & $60(6)$ & $61(56,65)$ \\
\hline \multicolumn{7}{|l|}{ Hospital Anxiety and Depression Scale } \\
\hline Total & & $14(7)$ & $12(9-18)$ & & $16(9)$ & $14(9-23)$ \\
\hline Anxiety & & $8(4)$ & $7(5-12)$ & & $9(5)$ & $8(6-11)$ \\
\hline Depression & & $5(3)$ & $5(4-6)$ & & $7(4)$ & $6(3-12)$ \\
\hline Rating Anxiety in Dementia & & $18(6)$ & $17(14-21)$ & & $21(6)$ & $22(17-24)$ \\
\hline Cornell Scale for Depression in Dementia & & $14(5)$ & $13(11-17)$ & & $18(7)$ & $19(13-22)$ \\
\hline \multicolumn{7}{|l|}{ Neuropsychiatric Inventory } \\
\hline Total & & $24(17)$ & $22(13-31)$ & & $28(12)$ & $27(22-39)$ \\
\hline Total carer distress & & $12(7)$ & $10(7-16)$ & & $14(6)$ & $13(9-18)$ \\
\hline \multicolumn{7}{|l|}{ Clinical Dementia Rating } \\
\hline Questionable/mild dementia & $21(84)$ & & & $21(84)$ & & \\
\hline Moderate dementia & $4 / 25(16)$ & & & $4 / 25(16)$ & & \\
\hline
\end{tabular}


Table 2 Baseline summary statistics for the carer by randomised group

\begin{tabular}{|c|c|c|c|c|c|c|}
\hline \multirow[b]{2}{*}{ Variable } & \multicolumn{3}{|c|}{ CBT group $(n=25)$} & \multicolumn{3}{|c|}{ TAU group $(n=25)$} \\
\hline & $n(\%)$ & Mean (s.d.) & Median (IQR) & $n(\%)$ & Mean (s.d.) & Median (IQR) \\
\hline \multicolumn{7}{|l|}{ Sociodemographics } \\
\hline Age & & & $69(62-80)$ & & & $66(51-74)$ \\
\hline Male & $11(44)$ & & & $9(36)$ & & \\
\hline Black or minority ethnicity & $0(0)$ & & & $0(0)$ & & \\
\hline \multicolumn{7}{|l|}{ Years in education } \\
\hline \multicolumn{7}{|l|}{ Relationship to participant } \\
\hline Spouse/partner & $18(72)$ & & & $11(44)$ & & \\
\hline Son/daughter & $7(28)$ & & & $9(36)$ & & \\
\hline Other & $0(0)$ & & & $5(20)$ & & \\
\hline Time spent as a carer (months) & & & $24(18-48)$ & & & $24(18-36)$ \\
\hline Hours a week spent caring & & & $61(10-168)$ & & & $15(6-80)$ \\
\hline \multicolumn{7}{|l|}{ Standardised scales } \\
\hline Quality of Life - Alzheimer's Disease & & $32(5)$ & $33(31-35)$ & & $32(6)$ & $32(27-37)$ \\
\hline Quality of Caregiver-Patient Relationship & & $57(7)$ & $57(54-61)$ & & $54(8)$ & $52(48-61)$ \\
\hline \multicolumn{7}{|l|}{ Hospital Anxiety and Depression Scale } \\
\hline Total & & $10(6)$ & $9(4-12)$ & & $9(5)$ & $9(6-13)$ \\
\hline Anxiety & & $6(4)$ & $6(2-8)$ & & $6(4)$ & $5(3-9)$ \\
\hline Depression & & $4(4)$ & $3(1-7)$ & & $4(2)$ & $4(2-4)$ \\
\hline
\end{tabular}

$16 \%$ on anxiolytic medication. Day services were used by $30 \%$ of all participants. Use of both antidepressant and anxiolytic medication was higher in the TAU group. There was no evidence that anyone was taking part in any other therapeutic intervention specifically for anxiety.

\section{Recruitment and retention}

Figure 1 shows the flow of participants through the trial. In total, 153 participants were referred to the study, of whom 93 came from secondary care services (memory clinics, Admiral Nursing), 26 through searches of case notes by the researcher, 22 from voluntary services, 7 via other research studies and 5 via other routes (for example self-referral). One hundred and three dyads were screened out as they did not meet eligibility criteria (63), refused participation (34), the person with dementia died (4) or became ill (2). Resulting in 50 participant-carer dyads randomised to either CBT plus TAU $(n=25)$ or TAU only $(n=25)$. Nine participants withdrew from the trial at first follow-up. Two participants were unable to be assessed at first follow-up but were assessed at second follow-up. Another three withdrew from the trial at second follow-up. Primary outcome data were available for all participants who were assessed at the first follow-up.

\section{Feasibility}

It was possible to recruit the required number within the given time frame (14 months), with approximately one in three referrals recruited into the trial. Attrition was acceptable: 39 of the 50 dyads were retained at 15 weeks and 38 at 6 months. Generally, people took up the intervention when offered it. Of the 25 participants allocated to the CBT group, 4 dropped out, 3 because the person or carer withdraw and 1 died. Of the remaining 21, 14 people attended all ten sessions. Seven people felt they had achieved their treatment goals sooner and finished early after six $(n=2)$, seven $(n=2)$, eight $(n=2)$ and nine sessions $(n=1)$. Of the 25 allocated to the TAU group, 8 dropped out of the research, 4 because of carer stressors, 2 because of dissatisfaction with allocation, 1 because of stress in the person with dementia and 1 where contact was lost. There was a significant difference in gender between those who were retained and dropped out. Of those retained,
$48 \%$ (19/30) were male and of those who dropped out, 9\% (1/11) were male. Thirty-six per cent of participants who dropped out (4/11) had moderate dementia, compared with $10 \%$ (4/39) of those who were retained. There were no reported adverse effects or side-effects of the intervention.

The trial therapists were asked to consider each person with dementia's 'suitability for cognitive therapy'. This provided a systematic approach to identifying those areas where 'pre-therapy' techniques may be required, for example strategies to expand emotional vocabulary or increase awareness of the link between cognitions, actions and emotions. Memory and language problems could be compensated for in most cases except where the degree of severity was such that the person with dementia was unable to 'hold in mind' the presence of the therapist during sessions and was continuously surprised by their presence, or in circumstances where the ability to have meaningful verbal exchanges was severely compromised. The therapy was least feasible in cases where there were significant and longstanding interpersonal difficulties between the person with dementia and their family carer or where there was no consistent family carer and the person with dementia needed a high level of in-session support.

\section{Adherence}

All four CBT therapists received a $2 \mathrm{~h}$ training session on the manual by G.C., a clinical psychologist with 15 years' experience of using CBT for older people. The same psychologist also provided clinical supervision. Sessions were recorded where possible and one session per dyad was coded by an independent psychologist for adherence to CBT using the Cognitive Therapy Scale - Revised (CTS-R). ${ }^{26}$ Ratings for six individuals were excluded because of requests not to be recorded $(n=4)$, technical problems $(n=1)$ and work primarily involving the carer $(n=1)$. Of the 15 recordings rated, an average score in the 'competent' range was achieved. There was a range in scores, largely because of the range of therapist expertise and extent to which participants met 'suitability for cognitive therapy' criteria.

\section{Main clinical outcome at 15 weeks}

Table 3 shows that, using the RAID scale, anxiety was significantly lower in the CBT group at 15 weeks $(-4.32,95 \%$ CI -8.21 to 


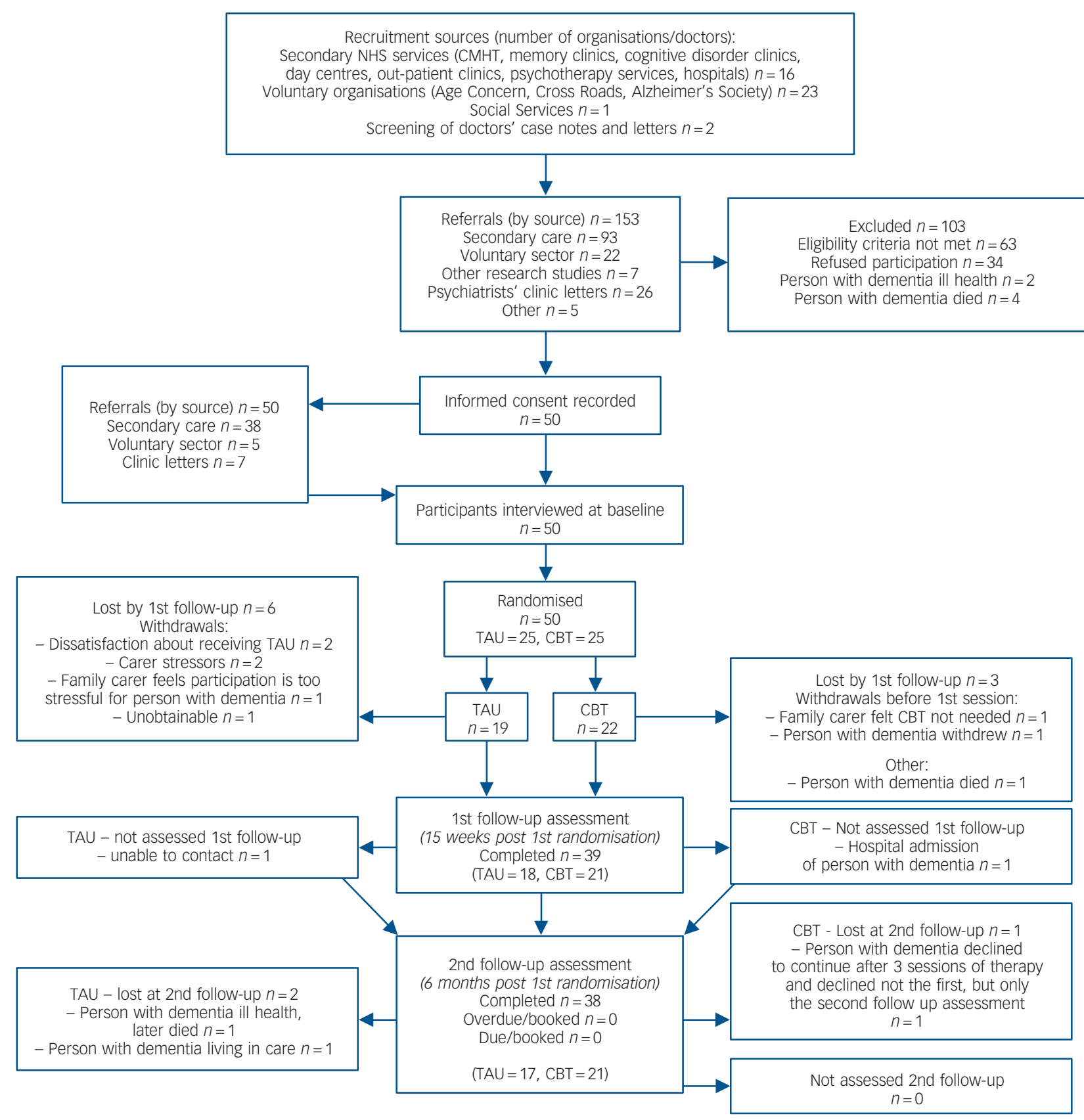

\section{Fig. 1 CONSORT diagram.}

NHS, National Health Service, CMHT, community mental health team; CBT, cognitive-behavioural therapy; TAU, treatment as usual.

-0.43). This lost statistical significance when adjusted for baseline anxiety and cognition $(-3.10,95 \% \mathrm{CI}-6.55$ to 0.34$)$.

\section{Secondary outcomes at 15 weeks}

Depression, as measured by the CSDD was also significantly lower in the CBT group and remained so following adjustment $(-5.37,95 \%$ CI -9.50 to -1.25$)$. However, we emphasise that because of the small sample we were not able to also adjust for baseline CSDD score in this analysis. There were no significant differences or notable trends in quality of life, cognition, anxiety and depression (measured by the HADS) or the quality of caregiver-patient relationship from the carer or patient perspective (Table 3 ).

\section{Longer-term outcomes}

The advantage shown by the CBT group over the TAU group with regard to anxiety was maintained at 6 months, with those in the CBT group scoring on average 4.59 points lower than those in the TAU group although this fell a little short of statistical significance after adjustment (95\% CI -9.34 to 0.15 ). The difference in depression score on the CSDD was similar to that at 15 weeks and was also statistically significant after adjustment $(-5.08,95 \%$ CI -9.25 to -0.92$)$. As at 15 weeks, there were no statistically significant differences in any other variables.

\section{Cost analysis}

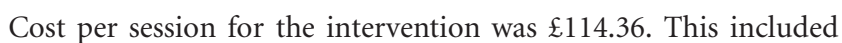
the average time spent by the therapist administering the 
Table 3 Outcomes at 15 weeks and 6 months, coefficients for cognitive-behavioural therapy

\begin{tabular}{|c|c|c|c|c|}
\hline \multirow[b]{2}{*}{ Outcome } & \multicolumn{2}{|c|}{ Unadjusted coefficient (95\% Cl) } & \multicolumn{2}{|c|}{ Adjusted coefficient $(95 \% \mathrm{Cl})^{\mathrm{a}}$} \\
\hline & 15 weeks & 6 months & 15 weeks & 6 months \\
\hline \multicolumn{5}{|l|}{ Participant } \\
\hline Rating Anxiety in Dementia & $-4.32(-8.21$ to -0.43$)$ & $-5.47(-10.67$ to -0.27$)$ & $-3.10(-6.55$ to 0.34$)$ & -4.59 ( -9.34 to 0.15$)$ \\
\hline Mini-Mental State Examination & $1.45(-2.58$ to 5.49$)$ & $0.86(-2.75$ to 4.48$)$ & $0.21(-1.72$ to 2.15$)$ & $-0.06(-1.94$ to 1.81$)$ \\
\hline Quality of Life - Alzheimer's Disease & $1.44(-2.24$ to 5.12$)$ & $-0.85(-4.49$ to 2.79$)$ & $0.70(-2.85$ to 4.26$)$ & $-0.90(-4.52$ to 2.71$)$ \\
\hline \multicolumn{5}{|l|}{ Hospital Anxiety and Depression Scale } \\
\hline Total & $-0.75(-5.09$ to 3.59$)$ & $-0.05(-5.60$ to 5.50$)$ & $0.02(-3.89$ to 3.94$)$ & $0.22(-4.88$ to 5.31$)$ \\
\hline Anxiety & $0.47(-1.89$ to 2.83$)$ & $0.30(-2.75$ to 3.35$)$ & $0.90(-1.10$ to 2.90$)$ & $0.43(-2.35$ to 3.21$)$ \\
\hline Depression & $-1.22(-3.96$ to 1.52$)$ & $-0.35(-3.56$ to 2.86$)$ & $-0.88(-3.63$ to 1.88$)$ & $-0.22(-3.33$ to 2.90$)$ \\
\hline Cornell Scale for Depression in Dementia & $-6.34(-10.60$ to -2.08$)$ & $-5.46(-9.62$ to -1.31$)$ & $-5.37(-9.50$ to -1.25$)$ & $-5.08(-9.25$ to -0.92$)$ \\
\hline \multicolumn{5}{|l|}{ Neuropsychiatric Inventory } \\
\hline Total & $-7.90(-18.43$ to 2.63$)$ & $-10.06(-20.63$ to 0.51$)$ & $-7.19(-18.21$ to 3.82$)$ & $-9.42(-20.10$ to 1.27$)$ \\
\hline Total carer distress & $-2.37(-6.81$ to 2.06$)$ & $-3.25(-8.41$ to 1.91$)$ & $-2.61(-7.18$ to 1.97$)$ & $-2.85(-8.06$ to 2.36$)$ \\
\hline \multicolumn{5}{|l|}{ Carer } \\
\hline Quality of Life - Alzheimer's Disease & $2.61(-1.52$ to 6.74$)$ & $2.41(-1.88$ to 6.69$)$ & $1.00(-1.85$ to 3.85$)$ & $1.08(-1.81$ to 3.97$)$ \\
\hline $\begin{array}{l}\text { Quality of Caregiver-Patient Relationship, total } \\
\text { Hospital Anxiety and Depression Scale }\end{array}$ & $3.88(-2.08$ to 9.85$)$ & $5.24(-0.39$ to 10.87$)$ & $-0.32(-4.88$ to 4.24$)$ & $1.27(-2.38$ to 4.92$)$ \\
\hline Total & $-0.60(-4.27$ to 3.06$)$ & $0.25(-3.78$ to 4.27$)$ & $0.07(-2.62$ to 2.76$)$ & $0.93(-2.06$ to 3.91$)$ \\
\hline Anxiety & $-0.80(-3.34$ to 1.74$)$ & $0.06(-2.68$ to 2.79$)$ & $-0.28(-2.01$ to 1.44$)$ & $0.70(-1.60$ to 2.99$)$ \\
\hline Depression & $0.20(-1.50$ to 1.90$)$ & $0.19(-2.11$ to 2.49$)$ & $0.38(-0.92$ to 1.67$)$ & $0.16(-1.64$ to 1.96$)$ \\
\hline
\end{tabular}

intervention (including planning), therapist training, travel and equipment. Average session attendance per person was 8.8 , hence

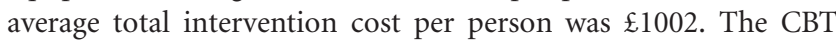
group had higher pre-baseline mean total cost from a health and social care perspective compared with TAU, with a mean difference of $£ 834.27$ (Table 4). Although this difference was not significant ( $95 \%$ bias-corrected CI $-£ 285.77$ to $£ 3069.38$ ), it needs to be taken into account when comparing post-randomisation costs, and was controlled for.

Table 5 shows costs incurred between baseline and follow-up 1. Although the costs (from a health and social care perspective) are significantly lower for the CBT group (unadjusted mean

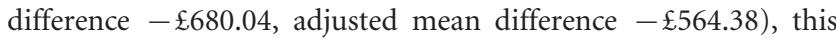
was not enough to offset the intervention cost. Including the cost of the intervention, total costs were higher for the CBT group compared with TAU, although this difference was not significant,

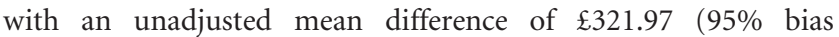

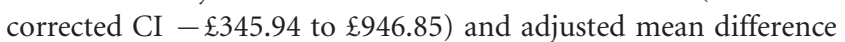
of $£ 769.80$ (95\% bias-corrected CI $-£ 121.99$ to $£ 1697.38$ ).

Between the first and second follow-up, mean costs were again higher for the CBT group compared with the TAU group, although this difference was not significant, with an unadjusted mean difference of $\mathfrak{E} 1085.02$ (95\% bias-corrected CI - $\mathfrak{2} 354.81$ to $£ 4078.64$ ), and an adjusted mean difference of $£ 256.12$ (95\% bias-corrected CI -599.05 to 1506.23) (Table 6). The first sensitivity analysis (which used no imputations) found no deviations from the main analysis with regard to trends or significance of any findings. The second sensitivity analysis (which removed high-cost outliers) found no significant difference in costs from a health and social care perspective at first follow-up, which was not surprising as sample size was reduced.

\section{Power calculation for a full trial}

For an unadjusted analysis, to detect a difference of four points on the RAID at 15 weeks (14 v. 18 in the CBT v. TAU groups, respectively), both with a standard deviation of 6 and $90 \%$ power, 48 people would be needed in each group to provide data on the RAID at the primary end-point (15 weeks).

\section{Discussion}

\section{Summary of results}

This trial demonstrated that formulation-based CBT is feasible for people with mild to moderate dementia and clinically significant anxiety. At 15 weeks, there were differences in anxiety that approached significance, and these improvements remained at 6 months. Although the CBT intervention was targeted at the thoughts, feelings and behaviours characteristic of anxiety, the more significant finding was the difference in depression as

Table 4 Participant pre-baseline costs ( $f$ ) by service group with mean imputations

\begin{tabular}{|c|c|c|c|}
\hline Service group & $\begin{array}{l}\text { CBT group, mean (s.d.) } \\
\qquad(n=25)\end{array}$ & $\begin{array}{l}\text { TAU group, mean (s.d.) } \\
\qquad(n=25)\end{array}$ & $\begin{array}{l}\text { Difference (unadjusted), mean } \\
\quad(95 \% \text { bias-corrected } \mathrm{Cl})\end{array}$ \\
\hline Accommodation & 492.77 (2349.54) & - & 492.77 (20.05 to 1706.41$)$ \\
\hline Hospital services & $614.32(2349.54)$ & $558.10(961.47)$ & $56.22(-435.97$ to 499.06$)$ \\
\hline Community services & $744.66(1458.53)$ & $565.47(715.84)$ & 179.19 ( -311.87 to 997.75$)$ \\
\hline Equipment/adaptations & $26.13(62.33)$ & $29.59(715.84)$ & $-3.47(-54.12$ to 35.33$)$ \\
\hline Day services & 88.79 (217.52) & $44.52(135.72)$ & $44.28(-52.80$ to 146.21$)$ \\
\hline Medication & $296.98(193.85)$ & $231.70(135.72)$ & $65.28(-31.04$ to 175.57$)$ \\
\hline Total (health and social care perspective) & 2263.65 (3937.53) & $1429.38(1342.21)$ & $834.27(-285.77$ to 3069.38$)$ \\
\hline
\end{tabular}




\begin{tabular}{|c|c|c|c|c|}
\hline Service group & $\begin{array}{l}\text { CBT group, } \\
\text { mean (s.d.) } \\
\quad(n=21)\end{array}$ & $\begin{array}{l}\text { TAU group, } \\
\text { mean (s.d.) } \\
(n=18)\end{array}$ & $\begin{array}{c}\text { Difference, unadjusted: } \\
\text { mean }(95 \% \\
\text { bias-corrected } \mathrm{Cl})\end{array}$ & $\begin{array}{c}\text { Difference, adjusted: }^{\text {a }} \\
\text { mean }(95 \% \\
\text { bias-corrected } \mathrm{Cl})\end{array}$ \\
\hline Accommodation & $17.85(81.79)$ & $48.58(206.12)$ & $-30.74(-140.22$ to 78.75$)$ & $-4.88(-96.86$ to 61.03$)$ \\
\hline Hospital services & $244.10(277.36)$ & $460.17(526.09)$ & $-216.07(-510.82$ to 36.41$)$ & $-146.34(-446.93$ to 55.91$)$ \\
\hline Community services & $321.60(427.14)$ & 767.40 (930.73) & $-445.80(-978.57$ to -2.00$)$ & $-417.19(-980.13$ to -95.35$)$ \\
\hline Equipment/adaptations & $20.94(76.73)$ & $32.30(90.14)$ & $-11.37(-64.85$ to 36.68$)$ & -11.84 ( -78.66 to 42.95$)$ \\
\hline Day services & $111.53(222.65)$ & $70.30(152.85)$ & $41.23(-80.77$ to 250.47$)$ & 20.15 ( -90.11 to 223.48$)$ \\
\hline Medication & $266.55(222.65)$ & 283.85 (176.08) & $-17.3(-128.48$ to 90.49$)$ & $-4.27(-115.26$ to 106.71$)$ \\
\hline Total (health and social care perspective) & $982.56(823.55)$ & $1662.61(1170.58)$ & $-680.04(-1401.91$ to -67.46$)$ & $-564.38(-1252.08$ to -112.85$)$ \\
\hline CBT intervention cost & $1002.01(222.65)$ & - & 1002.01 (892.01 to 1086.42 ) & 1010.96 (898.46 to 1102.99$)$ \\
\hline Health and social care plus CBT cost & $1984.58(222.65)$ & $1662.61(1170.58)$ & $321.97(-345.94$ to 946.85$)$ & $769.80(-121.99$ to 1697.38$)$ \\
\hline
\end{tabular}

measured by the CSDD at both 15- and 26-week follow-up. CBT led to a short-term reduction in health and social care costs (by 15 weeks), although this reduction was not enough to outweigh the cost of the intervention itself. In other words, CBT was cost-neutral. There were no significant changes in any other outcomes.

\section{Acceptability and feasibility of CBT}

The therapy was acceptable to people with dementia and their family carers as demonstrated by their willingness to participate, uptake of the intervention and low level of withdrawal from the intervention. The intervention was feasible for those with mild to moderate dementia (MMSE scores ranging from 25 to 16), although greater scaffolding by the therapist, a slower pace, greater repetition, increased emphasis on behavioural rather than cognitive techniques and a higher degree of involvement from family carers was necessary with people in the more moderate stages of dementia. Participants with dementia who were able to identify (a) unhelpful persistent negative automatic thoughts, conditional beliefs ('rules for living') or self-defeating cognitive processes (for example catastrophic thinking) and (b) more helpful alternative approaches, were also able to retain information and demonstrate the ability to 'stop, think and do differently' without necessarily needing prompts from family carers. In cases where the person was unable to engage in such processes, family carers who had engaged with the CBT rationale were able to support the person to apply coping statements and techniques such as distraction and relaxation. A challenge for this research was creating a manualised approach with enough built in flexibility to cover a variety of clinical presentations, both in terms of the profile of cognitive deficits and the nature and duration of the anxiety. One method for providing flexibility is to have a range of 'modules' within the manual, an approach used both here and in the 'peaceful mind' CBT studies in the USA. ${ }^{11,12}$

\section{Strengths and limitations}

There were several limitations to this study. First, there was a significant difference in baseline anxiety on the RAID scale, with the TAU group being significantly more anxious. It is therefore hard to know how effective CBT might have been for a more anxious group and one would hope for a more balanced sample in a larger trial. One therapist saw the majority of patients (18 participants), with only 4 participants seen by the three other therapists (one of whom treated 2 and two who treated 1 patient each). This could imply that the effects were largely as a result of the therapist rather than the intervention. However, the strength of this approach is that there was limited therapist variability, hence interpretations of the manual will have predominantly been the same.

There was no measureable impact of the therapy on anxiety measured using the HADS. This may be because of differences in content between the RAID and the HADS anxiety scale, or because of differences in methods of administration. Both the CSDD and the RAID scale take into account the carer's and rater's view of presenting symptomatology rather than relying on self-report by the person with dementia alone. Finally, in a full trial we could perform a full cost-effectiveness analysis, looking at trade-offs between better outcomes and higher costs. This was not feasible with this small sample pilot, which only considered costs from a health and social care perspective. It may be hypothesised,

\begin{tabular}{|c|c|c|c|c|}
\hline Service group & $\begin{array}{l}\text { CBT group, } \\
\text { mean (s.d.) } \\
\quad(n=21)\end{array}$ & $\begin{array}{l}\text { TAU group, } \\
\text { mean (s.d.) } \\
(n=17)\end{array}$ & $\begin{array}{c}\text { Difference, unadjusted: } \\
\text { mean }(95 \% \\
\text { bias-corrected } \mathrm{Cl})\end{array}$ & $\begin{array}{c}\text { Difference, adjusted: } \\
\text { mean }(95 \% \\
\text { bias-corrected } \mathrm{Cl})\end{array}$ \\
\hline Accommodation & $113.63(520.73)$ & - & 113.63 (76.98 to 280.74) & $19.08(-94.02$ to 129.03$)$ \\
\hline Hospital services & $448.84(650.05)$ & $296.68(345.75)$ & $152.16(-152.81$ to 493.88$)$ & $79.05(-175.83$ to 334.93$)$ \\
\hline Community services & $1396.37(4067.95)$ & $623.30(832.37)$ & $773.07(-401.88$ to 3117.64$)$ & $85.67(-627.76$ to 1102.29$)$ \\
\hline Equipment/adaptations & $2.34(6.51)$ & $3.80(7.66)$ & $-1.47(-5.74$ to 3.38$)$ & $-2.25(-6.42$ to 2.03$)$ \\
\hline Day services & 84.99 (144.45) & $61.29(84.25)$ & $23.70(-44.42$ to 105.85$)$ & $16.37(-55.33$ to 95.59$)$ \\
\hline Medication & $297.56(179.77)$ & $273.65(160.45)$ & $23.91(-92.28$ to 129.09$)$ & $58.20(-36.01$ to 151.23$)$ \\
\hline Total (health and social care perspective) & $2343.73(5072.26)$ & 1258.72 (971.47) & $1085.02(-354.81$ to 4078.64$)$ & $256.12(-599.05$ to 1506.23$)$ \\
\hline
\end{tabular}


for example, that CBT might lead to reduced carer costs if outcomes are better for the people with dementia. Our analysis of costs from a health and social care perspective was a strength in that it is of relevance to decision makers considering whether their organisation should implement CBT.

\section{Implications for research and practice}

The results suggest that a larger, fully powered RCT is now required to assess the effectiveness of CBT for anxiety in dementia. The data from this trial have been used to provide a power calculation for a full RCT, suggesting that a minimum of 96 participants (48 in each group) would be required prior to inflation for drop-out and additional inflation using the intraclass correlation associated with clustering by therapist. The manual is written for use by therapists who already have a good knowledge of using CBT and experience of work with people with dementia, although prior experience of carrying out CBT with people with dementia was not required. Future research may be required in evaluating the effectiveness of the therapy delivered by nonspecialists. The manual developed for this trial is now published, ${ }^{27}$ enabling others to use it. Our results on depression indicate further investigation of the effectiveness of CBT for depression in dementia is also warranted.

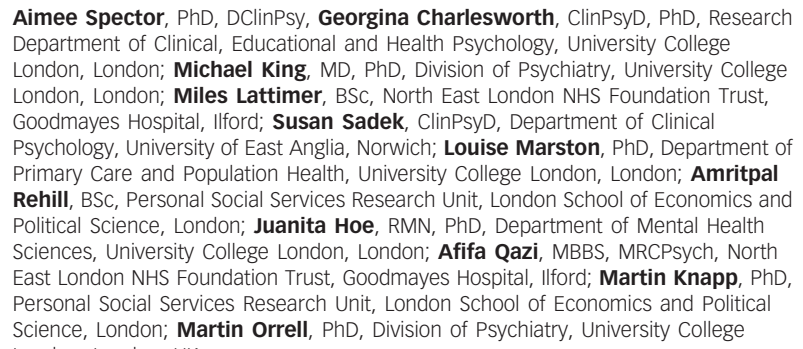

Correspondence: Aimee Spector, University College London, 1-19 Torrington Place, London WC1E 7HB, UK. Email: a.spector@ucl.ac.uk

First received 10 Oct 2013, final revision 18 Aug 2014, accepted 18 Sep 2014

\section{Funding}

This article presents independent research funded by the National Institute for Health Research (NIHR) under its Research for Patient Benefit (RfPB) Programme (grant reference number PB-PG-0609-18230). The views expressed are those of the author(s) and not necessarily those of the NHS, the NIHR or the Department of Health. The grant holders are A.S. (principal investigator), M.O., G.C., A.Q., M.K. and J.H.

\section{Acknowledgements}

PRIMENT Clinical Trials Unit at UCL (www.ucl.ac.uk/priment) provided general methodological advice at the design stage and throughout the trial, performed the randomisation and undertook analysis of the clinical outcomes. We would like to thank Kate Harwood, family carer, who provided support and a service-user perspective throughout the study; Dr Renee Romeo, London School of Economics, who provided additional support on the cost analysis and Nina Melunsky, who provided additional general support for the trial.

\section{References}

1 Porter V, Buxton WG, Fairbanks LA, Strickland T, O'Connor SM, et al. Frequency and characteristics of anxiety amongst patients with Alzheimer's disease and related dementias. J Neuropsychiatry Clin Neurosci 2003; 15 180-6.

2 Teri L, Logsdon R, Uomoto J, McCurry SM. Behavioural treatment of depression in dementia patients: a controlled clinical trial. J Gerontol 1999; 52: $159-66$.
3 Orrell M, Bebbington P. Psychosocial stress and anxiety in senile dementia. J Affect Disord 1996; 39: 165-73.

4 Moretti R, Torre P, Antonello R, Pizzolato G. Atypical neuroleptics as a treatment of agitation and anxiety in Alzheimer's disease: risks or benefits. Expert Rev Neurother 2006; 6: 705-10.

5 Beck AT, Clark DA. An information processing model of anxiety: automatic and strategic processes. Behav Res Ther 1997; 35: 49-58.

6 Stanley MA, Wilson NL, Novy DM, Rhoades HM, Wagener PD, Greisinger AJ, et al. Cognitive behavior therapy for generalized anxiety disorder among older adults in primary care - a randomized clinical trial. JAMA 2009; 301: 1460-7.

7 Department of Health. Improving Access to Psychological Therapies (IAPT). Department of Health, 2008.

8 Spector A, Thorgrimsen L, Woods B, Royan L, Davies S, Butterworth M, et al. Efficacy of an evidence-based cognitive stimulation therapy programme for people with dementia: randomised controlled trial. Br J Psychiatry 2003; 183: $248-54$.

9 Dagnan D, Chadwick P, Proudlove J. Toward an assessment of suitability of people with mental retardation for cognitive therapy. Cognit Ther Res 2000; 24: $627-36$.

10 Kipling $T$, Bailey $M$, Charlesworth $G$. The feasibility of a cognitive behavioral therapy group for men with mild to moderate cognitive impairment. Behav Cogn Psychother 1999; 27: 189-93.

11 Paukert AL, Calleo J, Kraus-Schuman C, Snow L, Wilson N, Petersen NJ, et al. Peaceful mind: an open trial of cognitive-behavioral therapy for anxiety in persons with dementia. Int Psychogeriatr 2010; 22: 1012-21.

12 Stanley MA, Calleo J, Bush AL, Wilson N, Snow AL, Kraus-Schuman C, et al. The peaceful mind program: a pilot test of a CBT-based intervention for anxious patients with dementia. Am J Geriatr Psychiatry 2013; 21: 696-708.

13 Craig P, Dieppe P, Macintyre S, Michie S, Nazareth I, Petticrew M, et al. Developing and evaluating complex interventions: the new Medical Research Council guidance. BMJ 2008; 337: a1655.

14 Spector $A$, Orrell $M$, Lattimer $M$, Hoe J, King $M$, Harwood $K$, et al. Cognitive behavioural therapy (CBT) for anxiety in people with dementia: study protocol for a randomised controlled trial. Trials 2012; 13: 197.

15 American Psychiatric Association. Diagnostic and Statistical Manual of Mental Disorder (4th edn) (DSM-IV). APA, 1994.

16 Hughes CP, Berg L, Danziger WL, Coben LA, Martin RL. A new clinical scale for the staging of dementia. Br J Psychiatry 1982; 140: 566-72.

17 Shankar KK, Walker M, Frost D, Orrell MW. The development of a valid and reliable scale for Rating Anxiety in Dementia (RAID). Aging Ment Health 1999; 3: 39-49.

18 Qazi A, Shankar K, Orrell M. Managing anxiety in people with dementia: a case series. J Affect Disord 2003; 76: 261-5.

19 Alexopoulos GS, Abrams RC, Young RC, Shamoian CA. Cornell Scale for Depression in Dementia. Biol Psychiatry 1998; 23: 271-284.

20 Zigmond AS, Snaith RP. "The Hospital Anxiety and Depression Scale". Acta Psychiatr Scand 1983; 67: 361-70.

21 Logsdon RG, Gibbons LE, McCurry SM, Teri L. Quality of life in Alzheimer's disease: patient and caregiver reports. J Ment Health Aging 1999; 5: 21-32.

22 cummings JL, Mega M, Gray K, Rosenberg-Thompson S, Carusi DA, Gornbein J, et al. The Neuropsychiatric Inventory: comprehensive assessment of psychopathology in dementia. Neurology 1994; 44: 2308-14.

23 Folstein MF, Folstein SE, McHugh PR. "Mini-mental state". A practical method for grading the cognitive state of patients for the clinician. J Psychiatr Res 1975; 12: 189-98.

24 Spruytte N, van Audenhove C, Lammertyn F, Storms G. The quality of caregiver and patient relationship in informal care for older adults with dementia and chronic psychiatric patients. Psychol Psychother 2002; 75 : 295-311.

25 Personal Social Services Research Unit. Unit Cost of Health and Social Care 2011. PSSRU, 2011 (http://www.pssru.ac.uk/archive/pdf/uc/uc2011/ uc2011.pdf)

26 Blackburn IM, James IA, Milne DL, Baker C, Standart S, et al. The Revised Cognitive Therapy Scale (CTS-R): Psychometric properties. Behav Cogn Psychother 2001; 29: 431-46.

27 Charlesworth G, Sadek S, Schepers A, Spector A. Cognitive behavior therapy for anxiety in people with dementia. A clinician guideline for a personcentered approach. Behav Modif 2014, Dec 16 (ePub ahead of print, doi: 10.1177/0145445514561317). 